\title{
THURSTONIAN MODELING OF RANKING DATA VIA MEAN AND COVARIANCE STRUCTURE ANALYSIS
}

\author{
Albert Maydeu-Olivares
}

\author{
UNIVERSITY OF ILLINOIS AT URBANA-CHAMPAIGN
}

\begin{abstract}
Although Thurstonian models provide an attractive representation of choice behavior, they have not been extensively used in ranking applications since only recently efficient estimation methods for these models have been developed. These, however, require the use of special-purpose estimation programs, which limits their applicability. Here we introduce a formulation of Thurstonian ranking models that turns an idiosyncratic estimation problem into an estimation problem involving mean and covariance structures with dichotomous indicators. Well-known standard solutions for the latter can be readily applied to this specific problem, and as a result any Thurstonian model for ranking data can be fitted using existing general purpose software for mean and covariance structure analysis. Although the most popular programs for covariance structure analysis (e.g., LISREL and EQS) cannot be presently used to estimate Thurstonian ranking models, other programs such as MECOSA already exist that can be straightforwardly used to estimate these models.
\end{abstract}

Key words: GMM, GLS, WLS estimation; UMD, ULS, EWMD estimation; permutation data; random utility models; structural equations models.

\section{Introduction}

In 1931, Thurstone suggested a class of models for fitting ranking data that has been highly influential in the literature (see Bock \& Jones, 1968) but not extensively used in applications since it requires the evaluation of multivariate normal integrals. With the development of efficient procedures for handling these integrals, over the past decade several approaches have been proposed to estimate Thurstonian ranking models (Böckenholt, 1993; Brady, 1989; Chan \& Bentler, 1998; Yao \& Böckenholt, 1999).

Thurstonian models can be expressed as a function of unobserved normal variates or as a function of differences of these variates. Under the first approach ranking probabilities are obtained by integrating a conic region, whereas under the second approach, these probabilities are obtained by integrating a parallelepiped (Takane \& de Leeuw, 1987). All the estimation procedures recently developed have adopted this second approach. Böckenholt (1993) used full information maximum likelihood to estimate these models. This approach requires evaluating numerically multivariate normal integrals, and thus it is not feasible for general covariance structures of the underlying normal variates when the number of objects is greater than five. This problem can be overcome using Bayesian resampling methods (Yao \& Böckenholt, 1999). However, resampling methods are still computationally too slow for practical applications of Thurstonian modeling.

An alternative solution to this problem is to employ a limited information estimator (Brady, 1989; Chan \& Bentler, 1998). Brady proposed estimating Thurstonian ranking models from binary or trinary rankings using a modification of the weighted minimum distance estimator proposed by Christoffersson (1975) for the normal ogive model. Just as Christofferson's estimator for the normal ogive model can be made computationally more efficient by making it two-stage

This paper is based on the author's doctoral dissertation. Ulf Böckenholt was my advisor. The author is indebted to Ulf Böckenholt for his comments on a previous version of this paper and to Gerhard Arminger for his extensive support on the use of MECOSA. The final stages of this research took place while the author was at the Department of Statistics and Econometrics, Universidad Carlos III de Madrid. Conversations with my colleague there, Adolfo Hernández, helped to greatly improve this paper.

Requests for reprints should be sent to Albert Maydeu-Olivares, Faculty of Psychology, University of Barcelona, P. Vall d'Hebron, 171, 08035 Barcelona, SPAIN. E-mail: amaydeu@psi.ub.es 
(Muthén, 1978), Chan and Bentler (1998) proposed a two-stage limited information estimator for Thurstonian models that uses information from trinary rankings. In the first stage, Chan and Bentler estimate an unrestricted Thurstonian model using either Lee, Poon and Bentler's (1995) partitioned maximum likelihood approach, or a direct weighted minimum distance approach as in Brady (1989). In a second stage, restricted Thurstonian models are estimated from the first stage results using a weighted minimum distance procedure.

Compared to full information maximum likelihood estimation, these limited information approaches are attractive because they have been found to provide comparable solutions (Böckenholt, 1993), they are computationally much less intensive, and they are able to handle larger models. However, existing limited information estimation procedures for Thurstonian ranking models present three problems:

1. Special purpose software is required.

2. Weighted minimum distance estimators are known to have a slow convergence to their asymptotic properties (Muthén \& Satorra, 1995) and may provide biased estimates in small samples (Clark, 1996).

3. The degrees of freedom available for testing must be determined at sample values. This is because there are systematic dependencies among the trinary rankings used as sample statistics. Consequently, their asymptotic covariance matrix will be singular yet its rank is presently unknown and it is evaluated using the data at hand.

Here we propose an alternative limited information approach to estimating Thurstonian ranking models that overcomes these three problems as follows:

1. We transform the observed ranking patterns to binary ranking patterns and formulate the model as a paired comparisons model as was first suggested by Thurstone (1931). As a result, these models can be straightforwardly fitted using existing software for mean and covariance structure analysis with dichotomous indicators.

2. We employ an unweighted minimum distance estimator as proposed by Muthén (1993) which provides unbiased estimates for covariance structures (Clark, 1996) and more accurate standard errors and model tests in small samples (Muthén, 1993).

3. Instead of binary or trinary rankings, we use as sample statistics choice proportions involving pairs, triplets and tetrads of objects. We have been able to determine the dependencies among these proportions, and therefore we provide the degrees of freedom available for modeling.

Thurstonian models are attractive because (a) they provide a rich representation of choice behavior that does not assume that objects are judged independently of each other, and (b) they can be given a straightforward substantive interpretation. However, in Thurstonian models identification issues need to be considered carefully to obtain an interpretable model, since the ease of interpretation of Thurstonian model parameters is easily lost when identification constraints are introduced to estimate them (Brady, 1989). Nonetheless, there are a number of members in the Thurstonian class of models (for an overview see Takane, 1987; and Böckenholt, 1992, 1993) and it is not feasible to consider meaningful identification restrictions for all of them in this paper. Consequently here we shall discuss detailed identification restrictions only for the unrestricted Thurstonian model. Our interest in this particular model arises from the fact that by fitting an unrestricted model one may test the suitability of the Thurstonian class to model ranking data, since any other Thurstonian model is just a restrictive version of this case.

This paper is structured as follows. First we shall review Thurstone's original ideas for the analysis of ranking data. Next we shall provide the two alternative formulations of Thurstonian ranking models as restricted multinomial models: as functions of normal variates or as functions of differences of normal variates. The latter, in turn, can be expressed in three different forms. One was used by Yao \& Böckenholt (1999), another was used by Böckenholt (1992) and Chan and Bentler (1998), and a third one, which is amenable to mean and covariance struc- 
tures analysis, will be provided here. This third approach stems directly from the formulation of Thurstonian modeling of paired comparisons data as a mean and covariance structures problem (Takane, 1987; see also Takane, 1989). Next, we shall discuss a set of identification restrictions for the unrestricted Thurstonian model that can be given a straightforward interpretation. Then, we shall discuss how these models can be estimated using sequential limited information estimators following Muthén (1978, 1993). Finally, an application involving fitting an unrestricted Thurstonian model and a one-factor model will be provided to illustrate the usefulness of this approach.

Most technical issues will be relegated to appendices. Appendix A provides the relationship between the expression for Thurstonian models employed in this paper and those given previously in the literature. Appendix B discusses alternative identification restrictions for the unrestricted Thurstonian model. Appendix C shows the asymptotic normality of Muthén's (1978) first stage estimates when applied to ranking data and provides the rank of their singular asymptotic covariance matrix.

\section{Thurstone's Model}

Consider a set of $n(n \geq 3)$ objects, $\left\{o_{1}, o_{2}, \ldots, o_{n}\right\}$ which are presented to a random sample of $N$ individuals from an homogeneous population. These subjects are asked to order the objects as a function of a given preference criterion. We may denote the ranking order provided by subject $j$ as $r_{j}=\left(r_{1 j}, r_{2 j}, \ldots, r_{n j}\right)$ where $r_{1 j}$ denotes which object in the original ordering of the objects was ranked first, $r_{2 j}$ denotes which object was ranked second, and so on. Since there are $n$ ! possible permutations of the original ordering of the objects, there are $n$ ! possible ranking patterns.

Let $\tilde{n}=\left(\begin{array}{c}n \\ 2\end{array}\right)$, then given the ranks, one can construct a dichotomous variable $y_{l}$ for each pairwise combination of objects $l=\left(i, i^{\prime}\right),\left(i=1, \ldots, n-1 ; i^{\prime}=i+1, \ldots, n\right)$, to indicate which object was ranked above the other

$$
y_{l}=\left\{\begin{array}{ll}
=1 & \text { if object } i \text { is ranked above object } i^{\prime} \\
=0 & \text { if object } i \text { is ranked below object } i^{\prime}
\end{array} \quad l=1, \ldots, \tilde{n}\right.
$$

Hence, one can express each $n$-dimensional ranking pattern $\mathbf{r}$ as an $\tilde{n}$-dimensional pattern of binary variables $\mathbf{y}$. An example of this mapping is provided in Table 1 .

Thurstone (1931) suggested modeling ranking data by applying his model for paired comparisons (Thurstone, 1927) to ranking data expressed as binary patterns. Thurstone's (1927) model for paired comparisons data assumes that for each pair of objects:

1. Each subject perceives the objects with continuous preferences, $t_{i j}$ and $t_{i^{\prime} j}, j=1, \ldots, N$.

2. The distribution of these unobserved preferences (denoted in Thurstone's terminology as discriminal processes) is bivariate normal in the population, $\left(\begin{array}{c}t_{i} \\ t_{i^{\prime}}\end{array}\right) \sim N_{2}\left(\left(\begin{array}{c}\mu_{i} \\ \mu_{i^{\prime}}\end{array}\right),\left(\begin{array}{cc}\sigma_{i}^{2} & \\ \sigma_{i i^{\prime}} & \sigma_{i^{\prime}}^{2}\end{array}\right)\right)$.

3. Choices are performed according to the following rule

$$
\text { choose object }\left\{\begin{array}{ll}
i & \text { if } \quad t_{i}>t_{i^{\prime}} \\
i^{\prime} & \text { if } \quad t_{i}<t_{i^{\prime}}
\end{array} .\right.
$$

The probability function of $y_{l}$ follows immediately

$$
\operatorname{Pr}\left(y_{l}=1\right)=\operatorname{Pr}\left(t_{i}>t_{i^{\prime}}\right)=\int_{-\infty}^{\infty} \int_{t_{i}}^{\infty} \phi\left(t_{i}, t_{i^{\prime}}: \mu_{i}, \mu_{i^{\prime}}, \sigma_{i}^{2}, \sigma_{i^{\prime}}^{2}, \sigma_{i i^{\prime}}\right) d t_{i} d t_{i^{\prime}},
$$

where $\phi(\cdot)$ denotes a normal density function, and obviously $\operatorname{Pr}\left(y_{l}=0\right)=1-\operatorname{Pr}\left(y_{l}=1\right)$. 

ates $t_{i}$

Thurstone (1927) proposed performing the following transformation on the random vari-

$$
y_{t}^{*}=t_{i}-t_{i^{\prime}} \quad l=\left(i, i^{\prime}\right) ; i=1, \ldots, n-1 ; i^{\prime}=i+1, \ldots, n .
$$

After this transformation we may express the preference rule (2) as a function of the $y_{l}^{*}$ random variables as follows

$$
\text { choose object } \begin{cases}i & \text { if } y_{l}^{*}>0 \\ i^{\prime} & \text { if } y_{l}^{*}<0\end{cases}
$$

Hence, instead of expressing the probability function of the dichotomous random variables $y_{l}$ as a function of $t_{i}$ and $t_{i^{\prime}}$, we may express it as function of their difference $y_{l}^{*}$ as

$$
\operatorname{Pr}\left(y_{l}=1\right)=\operatorname{Pr}\left(y_{l}^{*}>0\right)=\int_{0}^{\infty} \phi\left(y_{l}^{*}: \mu_{y_{l}^{*}}=\mu_{i}-\mu_{i^{\prime}}, \sigma_{y_{l}^{*}}^{2}=\sigma_{i}^{2}+\sigma_{i^{\prime}}^{2}-2 \sigma_{i i^{\prime}}\right) d y_{l}^{*} .
$$

We may perform a change of variable of integration in (6) and standardize each of the $y_{l}^{*}$ using $z_{l}=\frac{y_{l}^{*}-\mu_{y_{l}^{*}}}{\sigma_{y_{l}^{*}}}$. Then, $d y_{l}^{*}=\sigma_{y_{l}^{*}} d z_{l}$, and at $y_{l}^{*}=0, z_{l}=\frac{-\mu_{y_{l}^{*}}}{\sigma_{y_{l}^{*}}}$. Hence, we may rewrite $(6)$ as

$$
\operatorname{Pr}\left(y_{l}=1\right)=\operatorname{Pr}\left(z_{l}>0\right)=\int_{q_{t}}^{\infty} \phi\left(z_{l}: 0,1\right) d z_{l}=1-\Phi\left(q_{l}\right)=\Phi\left(-q_{l}\right),
$$

where $\Phi(\cdot)$ denotes the cumulative standard normal distribution function, and

$$
q_{l}=\frac{-\mu_{y_{l}^{*}}}{\sigma_{y_{l}^{*}}}=-\frac{\mu_{i}-\mu_{i^{\prime}}}{\left(\sigma_{i}^{2}+\sigma_{i^{\prime}}^{2}-2 \sigma_{i i^{\prime}}\right)^{1 / 2}} .
$$

Thurstone (1927) proposed estimating the model parameters, $\mu_{t}$ and $\Sigma_{t}$, by solving the system of equations consisting of

$$
p_{l}=\Phi\left(\frac{\mu_{i}-\mu_{i^{\prime}}}{\left(\sigma_{i}^{2}+\sigma_{i^{\prime}}^{2}-2 \sigma_{i i^{\prime}}\right)^{1 / 2}}\right) \quad l=1, \ldots, \tilde{n}
$$

where $p_{l}$ is the observed proportion of subjects preferring object $i$ over object $i^{\prime}$. Since there are only $\left(\begin{array}{l}n \\ 2\end{array}\right)=\frac{n(n-1)}{2}$ observed proportions $p_{l}$, the $n+\frac{n(n+1)}{2}$ parameters of the model cannot be estimated without further constraints (Thurstone, 1927). Consequently, he suggested two sets of constrains that identify the model: Case III in which $\Sigma_{t}$ is a diagonal matrix, and Case V in which $\Sigma_{t}=\sigma \mathbf{I}$. Because there are a number of constraints that can be imposed on $\mu_{t}$ and $\Sigma_{t}$ we speak of a class of models characterized by Assumptions 1 through 3 above. This paper focuses on one specific model in this class, the unrestricted Thurstonian model. By this we mean a model where $\mu_{t}$ and $\Sigma_{t}$ are unconstrained except for minimal restrictions needed for its identification.

\section{Sampling Theory}

Let $\left\{\mathbf{r}_{1}, \mathbf{r}_{2}, \ldots, \mathbf{r}_{n}\right.$ ! $\}$ denote the $n$ ! possible ranking patterns, and $\left\{m_{1}, m_{2}, \ldots, m_{n} !\right\}$ be the number of subjects with each pattern observed in the sample. Clearly, the joint distribution of the random variables $\left\{m_{1}, m_{2}, \ldots, m_{n}\right\}$ is multivariate hypergeometric. Yet as the size of the population becomes increasingly large relative to $N$, and $\frac{m}{N}=\pi$ remains constant, their joint distribution will be well approximated by a multinomial distribution. In this paper we shall assume that the multinomial approximation is appropriate and discuss what restrictions Thurstonian models for ranking data impose on multinomial probabilities. 
When the ranking patterns are expressed as patterns of binary variables $\mathbf{y}$, the $N$ observed patterns can be placed in a $2^{\tilde{n}}$ contingency table. The observed proportions $p_{l}$ in (8) are the first order marginals of this table. These are the sample statistics used in Brady's (1989) binary ranking estimator. Clearly, only a very limited amount of the information available in the data is employed by estimation methods based on these proportions alone. Furthermore, models such as the unrestricted Thurstonian model that are not identified from the first order marginals of this table only may be identified as soon as the second order marginals are considered. Second order marginal proportions, $p_{l l^{\prime}}$, involve triplets of objects, and as soon as $n>3$, also tetrads of objects. To see this, let $n=4$. In this case, $p_{21}$ is the sample proportion of $\left(\left(o_{1}>o_{3}\right) \cap\left(o_{1}>o_{2}\right)\right)$ but $p_{43}$ corresponds to $\left(\left(o_{2}>o_{3}\right) \cap\left(o_{1}>o_{4}\right)\right)$.

\section{Thurstone's Model Revisited}

The restrictions imposed by Thurstonian models on multinomial probabilities can be formulated in two different ways (Takane $\&$ de Leeuw, 1987), depending on whether these probabilities are expressed as a function of the normal variates $\mathbf{t}$, as in (3), or of their pairwise differences $\mathbf{y}^{*}$ as in (6).

\subsection{Thurstonian Models for Ranking Data Expressed as Functions of the Normal Variates $\mathbf{t}$}

Let $\mathbf{t}$ be an $n$-dimensional vector of continuous preferences, $\mathbf{t} \sim N_{n}\left(\mu_{t}, \Sigma_{t}\right)$, and let the ranking provided by subject $j$ be represented by an $\tilde{n}$-dimensional vector of binary variables $\mathbf{y}_{j}$. In this case, the probability of observing $\mathbf{y}_{j}$ can be expressed as

$$
\operatorname{Pr}\left(\mathbf{y}_{j}\right)=\int \underset{\overrightarrow{\mathbf{R}}}{ } \int \phi\left(\mathbf{t}: \boldsymbol{\mu}_{t}, \Sigma_{t}\right) d \mathbf{t}, \quad j=1, \ldots, N
$$

where $\breve{\mathbf{R}}$ is an $n$-dimensional area of integration where the following inequalities take place simultaneously $\left\{\begin{array}{lll}t_{i j}>t_{i^{\prime} j} & \text { if } & y_{l j}=1 \\ t_{i j}<t_{i^{\prime} j} & \text { if } & y_{l j}=0\end{array}\right.$.

To obtain the limits of integration in (9) we need to permute the objects according to the ranking order provided by each individual, so that the $i$ th interval of $\breve{R}$ is given by

$$
\breve{R}_{i}=\left\{\begin{array}{ll}
(-\infty, \infty) & \text { if } t_{r_{i}} \\
\left(t_{r_{i}-1}, \infty\right) & \text { otherwise }
\end{array} .\right.
$$

Therefore,

$$
\begin{aligned}
\operatorname{Pr}\left(\mathbf{y}_{j}\right) & \equiv \operatorname{Pr}\left(\mathbf{r}_{j}\right)=\operatorname{Pr}\left\{r_{1 j}, r_{2 j}, \ldots, r_{n j}\right\} \\
& =\int_{-\infty}^{\infty} \int_{t_{r_{1}}}^{\infty} \cdots \int_{t_{r_{n-2}}}^{\infty} \int_{t_{r_{n-1}}}^{\infty} \phi\left(\mathbf{t}: \mu_{t}, \Sigma_{t}\right) d t_{r_{n}} d t_{r_{n-1}} \cdots d t_{r_{2}} d t_{r_{1}}
\end{aligned}
$$

\subsection{Thurstonian Models for Ranking Data Expressed as Functions of Differences of Normal Variates $\mathbf{y}^{*}$}

In this case, the probability of subject $j^{\prime}$ s ranking pattern can be expressed as

$$
\operatorname{Pr}\left(\mathbf{y}_{j}\right)=\int \ldots \int \phi\left(\mathbf{y}^{*}: \mu_{y^{*}}=\mathbf{A} \mu_{t}, \Sigma_{y^{*}}=\mathbf{A} \Sigma_{t} \mathbf{A}^{\prime}\right) d \mathbf{y}^{*}
$$


where $\mathbf{R}$ is an $\tilde{n}$-dimensional area of integration obtained by the product of the intervals

$$
R_{l}= \begin{cases}(0, \infty) & \text { if } y_{l}=1 \\ (-\infty, 0) & \text { if } y_{t}=0\end{cases}
$$

and $\mathbf{A}$ is an $\tilde{n} \times n$ linear transformation matrix such that

$$
\mathbf{y}^{*}=\mathbf{A} \mathbf{t},
$$

where $a_{l k}=\left\{\begin{array}{rl}0 & \text { if } k \notin\left\{i, i^{\prime}\right\} \\ 1 & \text { if } k=i \\ -1 & \text { if } k=i^{\prime}\end{array} \quad, l=\left(i, i^{\prime}\right) ; i=1, \ldots, n-1 ; i^{\prime}=i+1, \ldots, n ; k=1, \ldots, n\right.$; so that for example, when $n=4$, A will be the following $6 \times 4$ matrix

$$
\mathbf{A}=\left[\begin{array}{rrrr}
1 & -1 & 0 & 0 \\
1 & 0 & -1 & 0 \\
1 & 0 & 0 & -1 \\
0 & 1 & -1 & 0 \\
0 & 1 & 0 & -1 \\
0 & 0 & 1 & -1
\end{array}\right]
$$

Matrix $A$ is of rank $n-1$, as can be readily verified by noting that $\mathbf{N}_{A}=\mathbf{1}_{n}^{\prime}$ is a basis for its null space. Now, when $\Sigma_{t}$ is positive definite it can be factored as $\boldsymbol{\Sigma}_{t}=\mathbf{F} \mathbf{F}^{\prime}$ with $r(\mathbf{F})=n$. Since $r\left(\Sigma_{y^{*}}\right) \equiv r\left(\left(\mathbf{A F}\left(\mathbf{F}^{\prime} \mathbf{A}^{\prime}\right)\right)=r(\mathbf{A F})\right.$, and $r(\mathbf{A F})=r(\mathbf{A})$ (Rao, 1973: p. 30), if follows that $r\left(\Sigma_{y^{*}}\right)=n-1$. Hence, $\phi\left(\mathbf{y}^{*}\right)$ is a singular multivariate normal density.

Equations (11) and (12) provide the two alternative formulations of Thurstone's (1931) ranking model. In the first one, ranking probabilities are obtained by integrating an $n$-dimensional multivariate normal density over a conic region. In the second one, ranking probabilities can be obtained by integrating an $n-1$ parallelepiped as shown in Appendix A.

The formulation of Thurstone's ranking model as a function of differences of normal variates, $\mathbf{y}^{*}$, can be readily estimated via mean and correlation structure analysis for dichotomous variables subject to suitable identification restrictions. These are discussed in the next section.

\section{Identification Restrictions for the Unrestricted Thurstonian Model for Ranking Data}

We shall consider two sets of identification restrictions: (a) identification restrictions for estimating the reduced form parameters of the model, $\boldsymbol{\mu}_{y^{*}}$ and $\boldsymbol{\Sigma}_{y^{*}}$, from the patterns of binary choices $\mathbf{y}$; and (b) identification restrictions for estimating the Thurstonian model parameters from the reduced form parameters.

Regarding the first set of identification restrictions, since the observed variables are dichotomous, the means and variances of $\mathbf{y}^{*}$ are not identifiable separately. This can be solved for instance by setting $\sigma_{y^{*}}^{2}=l, \forall l$, so that $\mathbf{y}^{*}$ has a correlation structure. The variance normalization restriction on $\mathbf{y}^{*}$ can be enforced by the transformation $\mathbf{z}^{*}=\mathbf{D} \mathbf{y}^{*}$, where $\mathbf{D}=\operatorname{diag}\left(\Sigma_{y^{*}}\right)^{-1 / 2}$, so that $\mathbf{z}^{*} \sim N\left(\boldsymbol{\mu}_{z^{*}}=\mathbf{D A} \boldsymbol{\mu}_{t}, \mathbf{P}_{z^{*}}=\mathbf{D A} \Sigma_{t} \mathbf{A}^{\prime} \mathbf{D}\right)$ has the required correlation structure.

Regarding the second set of identification conditions, since $\mathbf{A}$ is of rank $n-1$, the model parameters suffer from a location indeterminacy. In the mean structure, this indeterminacy can be solved by fixing one of the means, say $\mu_{n}=0$. In the correlation structure of the model, this location indeterminacy results in $\Sigma_{t}$ being only estimable under arbitrary restrictions among its elements (see Appendix B). This causes a serious problem when interpreting the model. A somewhat better solution is to estimate $\mathbf{P}_{t}$, the correlation matrix among the normal variates $\mathbf{t}$, instead of $\Sigma_{t}$. This amounts to identifying the model by setting $\sigma_{i i}=1, \forall i$ (Dansie, 1986).

These restrictions do not suffice to identify the model since $\mathbf{z}^{*}$ is insensitive to scale changes in $\mathbf{t}$ of the type $\mathbf{t}^{*}=c t$, where $c$ is any positive constant, since in this case, $\mu_{t^{*}}=c \mu_{t}$, 
$\mathbf{P}_{t^{*}}=c^{2} \mathbf{P}_{t}$, but because $\mathbf{D}_{t^{*}}=c^{-1} \mathbf{D}_{t}, \boldsymbol{\mu}_{z^{*}}$ and $\mathbf{P}_{z^{*}}$ would remain unchanged (Chan \& Bentler, 1998). Hence one additional parameter must be fixed to resolve this indeterminacy and identify the model. Since we are already fixing $\boldsymbol{\mu}_{n}=0$, fixing an additional parameter in $\boldsymbol{\mu}_{t}$ could result in serious convergence problems during estimation unless this value is very close to the parameter's true value. To avoid this problem, we shall fix instead one element of $\mathbf{P}_{t}$ equal to zero, say $\rho_{n, n-1}$.

In sum, with these identification restrictions we may rewrite (12) as

$$
\operatorname{Pr}\left(\mathbf{y}_{j}\right)=\int \ldots \int \phi\left(\mathbf{z}^{*}: \boldsymbol{\mu}_{z^{*}}=\mathbf{D A} \mu_{t}, \mathbf{P}_{z^{*}}=\mathbf{D A} \mathbf{P}_{t} \mathbf{A}^{\prime} \mathbf{D}\right) d \mathbf{z}^{*}
$$

where $\mu_{n}$ and $\rho_{n, n-1}$ are fixed parameters, and $\mathbf{D}=\operatorname{diag}\left(\mathbf{A} \mathbf{P}_{t} \mathbf{A}^{\prime}\right)^{-1 / 2}$. This model cannot be identified from first order information alone as there are more parameters, $\left(n^{2}+n-4\right) / 2$, than nonredundant binary choices. With these identification restrictions, as $\mu_{i}>0$ indicates a higher mean preference for object $i$ than for object $n$, the model assigns a higher probability to those ranking patterns beginning with object $i$ than to those beginning with object $n$. Similarly, as $\rho_{i, i^{\prime}}>0$ indicates a positive association between objects $i$ and $i^{\prime}$, the model assigns a higher probability to those ranking patterns in which objects $i$ and $i^{\prime}$ are together than to those in which these objects are apart (Dansie, 1986).

As for identification conditions for restricted Thurstonian models, we shall distinguish between models that can be expressed as restricting $\boldsymbol{\mu}_{t}$ and $\mathbf{P}_{t}$ and models that cannot. Typical examples of the latter are Case III and Case V models. A sufficient condition for the first set of models is that their parameters can be identified from the identified parameters of $\boldsymbol{\mu}_{t}$ and $\mathbf{P}_{t}$. To illustrate this point, consider the one-factor model in which $P_{t}=\lambda \boldsymbol{\lambda}^{\prime}+\Psi$ where $\boldsymbol{\Psi}$ is a diagonal matrix. This model can be identified by letting $\mu_{n}=0, \lambda_{n}=0$, and $\boldsymbol{\Psi}=\mathbf{I}-\operatorname{diag}\left(\boldsymbol{\lambda} \boldsymbol{\lambda}^{\prime}\right)$. Regarding the second set of models, one must assess whether their parameters are identified from the reduced-form parameters. Computer algebra (e.g., Bekker, Merckens, \& Wansbeek, 1994) is particularly well suited to this purpose. For instance, one can easily verify that the Case V model can be identified by letting $\mu_{n}=0$ and $\sigma=1$, whereas the Case III model can be identified by letting $\mu_{n}=0$ and $\sigma_{n}=1$.

\section{Estimation}

We shall estimate Thurstonian ranking models using a two stage limited information estimator. In a first stage, the reduced form parameters of the model $\boldsymbol{\mu}_{z^{*}}$ and $\mathbf{P}_{z^{*}}$ are estimated sequentially from the first and second order marginals of the $2^{\tilde{n}}$ contingency table as in Muthén (1978). In a second stage, the parameters of any Thurstonian ranking model, $\theta$, are estimated as the solution to

$$
\hat{F}_{W M D}=\min _{\theta}(\hat{\boldsymbol{\kappa}}-\boldsymbol{\kappa}(\boldsymbol{\theta})) \widehat{\boldsymbol{\Gamma}}^{-}(\hat{\boldsymbol{\kappa}}-\boldsymbol{\kappa}(\boldsymbol{\theta})),
$$

where $\boldsymbol{\kappa}=\left(\boldsymbol{\mu}_{z^{*}}, \operatorname{vecr}\left(\mathbf{P}_{z^{*}}\right)\right)^{\prime}$ is the vector of reduced form parameters of the model and $\boldsymbol{\Gamma}$ is the singular asymptotic covariance matrix of $\sqrt{N}(\hat{\kappa}-\kappa(\theta))$. Vecr( $(\cdot)$ is used here to denote an operator that stacks all lower diagonal elements of a matrix, excluding the diagonal, onto a column vector. Muthén (1978) first proposed this sequential limited information estimator for the multidimensional normal ogive model with $\mu_{z^{*}}=\tau$, the vector of thresholds of the normal ogive model.

For nonsingular $\boldsymbol{\Gamma}$ and under correct model specification, the resulting estimator $\hat{\theta}$ is consistent, asymptotically normal and asymptotically efficient among all estimators that use first and second order information (Muthén, 1978). Also, $N \hat{F}_{W M D}$ is asymptotically distributed as a central chi-square with $(v-q)$ degrees of freedom, where $v$ is the rank of $\Gamma$ and $q$ is the number of parameters of the model. Hence, $N \hat{F}_{W M D}$ can be used to test the restrictions imposed by the model on the reduced form parameters. These results also hold for singular $\Gamma$ (e.g., Satorra, 1989). 
Asymptotically optimal minimum distance estimators such as (16) have a slow convergence to their asymptotic distribution (Muthén \& Satorra, 1995). As proposed by Muthén (1993), an unweighted second stage minimum distance estimator

$$
\hat{F}_{U M D}=\min _{\theta}(\hat{\kappa}-\kappa(\theta))^{\prime}(\hat{\kappa}-\kappa(\theta))
$$

may show better small sample properties, specially for large models. Since $N \hat{F}_{U M D}$ is not asymptotically chi-squared, we shall use Satorra and Bentler's (1988) scaled and adjusted test statistics to test the fit of the UMD solution to the reduced form parameters.

The asymptotic properties of the second stage estimators rely on the asymptotic normality of the first stage estimator. In Appendix $C$ we show that $\hat{\kappa}$ is asymptotically normal when obtained from a binary contingency table arising from a ranking experiment, where there are $2^{\tilde{n}}-n$ ! structural zeroes. We also establish that the rank of $\Gamma$ in this case is

$$
\nu=\operatorname{dim}(\hat{\kappa})-\sum_{x=2}^{n-1}\left(\begin{array}{l}
x \\
2
\end{array}\right)=\frac{1}{2}\left(\left(\begin{array}{l}
n \\
2
\end{array}\right)^{2}+\left(\begin{array}{l}
n \\
2
\end{array}\right)\right)-\sum_{x=2}^{n-1}\left(\begin{array}{l}
x \\
2
\end{array}\right)
$$

\section{Application}

We asked 279 Spanish college students to rank four compact cars $\{1=$ Ford Fiesta, $2=$ Opel Corsa, 3 = Peugeot 106, $4=$ Wolkswagen Polo according to their purchase preferences. In Table 1 we provide the ranking patterns' observed frequencies in this sample. The unrestricted

TABLE 1.

\begin{tabular}{|c|c|c|c|c|}
\hline $\begin{array}{c}\text { binary ranking } \\
\text { patterns }(y)\end{array}$ & $\begin{array}{c}\text { ranking } \\
\text { patterns }(\mathbf{r})\end{array}$ & $\begin{array}{l}\text { observed } \\
\text { freq. }\end{array}$ & $\begin{array}{c}\text { expected } \\
\text { freq. (WMD) } \\
\end{array}$ & $\begin{array}{c}\text { expected } \\
\text { freq. (UMD) } \\
\end{array}$ \\
\hline 111111 & 1234 & 16 & 15.41 & 17.14 \\
\hline 111110 & 1243 & 16 & 8.74 & 10.76 \\
\hline 111011 & 1324 & 14 & 14.66 & 14.62 \\
\hline 111001 & 1342 & 2 & 6.79 & 6.54 \\
\hline 111100 & 1423 & 4 & 7.46 & 8.69 \\
\hline 111000 & 1432 & 9 & 6.12 & 6.16 \\
\hline 011111 & 2134 & 16 & 18.48 & 17.71 \\
\hline 011110 & 2143 & 10 & 11.03 & 11.47 \\
\hline 001111 & 2314 & 19 & 14.58 & 13.36 \\
\hline 000111 & 2341 & 3 & 5.05 & 5.17 \\
\hline 010110 & 2413 & 8 & 9.64 & 9.10 \\
\hline 000110 & 2431 & 9 & 5.42 & 5.28 \\
\hline 101011 & 3124 & 22 & 21.21 & 19.80 \\
\hline 101001 & 3142 & 14 & 10.17 & 9.57 \\
\hline 001011 & 3214 & 14 & 17.43 & 17.39 \\
\hline 000011 & 3241 & 3 & 5.94 & 7.03 \\
\hline 100001 & 3412 & 8 & 9.58 & 9.87 \\
\hline 000001 & 3421 & 11 & 6.55 & 8.03 \\
\hline 110100 & 4123 & 21 & 17.08 & 16.66 \\
\hline 110000 & 4132 & 12 & 13.85 & 12.34 \\
\hline 010100 & 4213 & 14 & 17.92 & 16.51 \\
\hline 000100 & 4231 & 9 & 10.39 & 10.28 \\
\hline 100000 & 4312 & 11 & 14.79 & 13.86 \\
\hline 000000 & 4321 & 14 & 10.64 & 11.60 \\
\hline
\end{tabular}

Observed and expected frequencies of ranking patterns in the compact cars data

Notes: 1 = Ford Fiesta, $2=$ Opel Corsa, $3=$ Peugeot 106, $4=$ Volkswagen Polo 
TABLE 2 .

Results of estimating an unrestricted Thurstonian model

\begin{tabular}{|c|c|c|c|c|c|}
\hline \multirow[b]{2}{*}{$\mathbf{P}_{t}$} & \multicolumn{4}{|c|}{ Parameter estimates and asymptotic standard errors } & \multirow[b]{2}{*}{$\mu_{t}$} \\
\hline & $\begin{array}{l}\text { Ford } \\
\text { Fiesta }\end{array}$ & $\begin{array}{l}\text { Opel } \\
\text { Corsa }\end{array}$ & $\begin{array}{c}\text { Peugeot } \\
106\end{array}$ & $\begin{array}{l}\text { Volkswagen } \\
\text { Polo }\end{array}$ & \\
\hline $\begin{array}{l}\text { Ford } \\
\text { Fiesta }\end{array}$ & 1 & $\begin{array}{c}0.66 \\
(0.07)\end{array}$ & $\begin{array}{c}0.59 \\
(0.08)\end{array}$ & $\begin{array}{c}0.19 \\
(0.09)\end{array}$ & $\begin{array}{c}0.14 \\
(0.09)\end{array}$ \\
\hline $\begin{array}{l}\text { Opel } \\
\text { Corsa }\end{array}$ & $\begin{array}{c}0.65 \\
(0.07)\end{array}$ & 1 & $\begin{array}{c}0.47 \\
(0.09)\end{array}$ & $\begin{array}{c}0.12 \\
(0.10)\end{array}$ & $\begin{array}{c}0.10 \\
(0.09)\end{array}$ \\
\hline $\begin{array}{l}\text { Peugeot } \\
106\end{array}$ & $\begin{array}{c}0.49 \\
(0.09)\end{array}$ & $\begin{array}{c}0.43 \\
(0.10)\end{array}$ & 1 & $\begin{array}{c}0 \\
\text { fixed }\end{array}$ & $\begin{array}{c}0.08 \\
(0.09)\end{array}$ \\
\hline $\begin{array}{l}\text { Volkswagen } \\
\text { Polo }\end{array}$ & $\begin{array}{c}0.16 \\
(0.10)\end{array}$ & $\begin{array}{c}0.12 \\
(0.11)\end{array}$ & $\begin{array}{c}0 \\
\text { fixed }\end{array}$ & 1 & $\begin{array}{c}0 \\
\text { fuxed }\end{array}$ \\
\hline$\mu_{t}$ & $\begin{array}{c}0.16 \\
(0.09)\end{array}$ & $\begin{array}{c}0.11 \\
(0.09)\end{array}$ & $\begin{array}{c}0.09 \\
(0.10)\end{array}$ & $\begin{array}{c}0 \\
\text { fixed }\end{array}$ & \\
\hline
\end{tabular}

Goodness of fit indices

\begin{tabular}{|c|c|c|c|c|c|c|}
\hline \multirow[b]{2}{*}{ Estimator } & \multicolumn{2}{|c|}{$H_{0}:\left\{\begin{array}{l}\boldsymbol{\mu}_{z^{*}}=\boldsymbol{\mu}_{z^{*}}(\boldsymbol{\theta}) \\
\mathbf{P}_{z^{*}}=\mathbf{P}_{z^{*}}(\boldsymbol{\theta})\end{array}\right.$} & \multicolumn{2}{|c|}{$H_{0}: \pi=\pi(\theta)$} & $d f=15$ & \multirow[b]{2}{*}{$p$-value } \\
\hline & Test statistic & $p$-value & $X^{2}$ statistic & $p$-value & $G^{2}$ statistic & \\
\hline WMD & $N \hat{F}=19.8$ & 0.02 & 28.76 & 0.02 & 28.55 & 0.02 \\
\hline UMD & $\begin{aligned} N \hat{F} & =13.26 \\
T_{s} & =18.14 \\
T_{a} & =13.27\end{aligned}$ & $\begin{array}{l}- \\
0.03 \\
0.05\end{array}$ & 25.55 & 0.04 & 26.74 & 0.03 \\
\hline
\end{tabular}

Notes: WMD estimates above the diagonal; UMD estimates below the diagonal; $T_{a}$ and $T_{s}$ are Satorra and Bentler's (1988) adjusted and scaled test statistics. The degrees of freedom for $T_{a}$ are 6.58 .

Thurstonian model (15) was fitted to these data using a WMD estimator as in Muthén (1978), and then using an UMD estimator as in Muthén (1993). The resulting model parameters, asymptotic standard errors and goodness of fit tests are shown in Table 2 . As can be seen in this table, given the identification restrictions $\rho_{43}=0$ and $\mu_{4}=0$, the estimated standard errors indicate that there are significant positive associations among the preferences for the Ford Fiesta, Opel Corsa and Peugeot 106, whereas the preferences for the Volkswagen Polo are unrelated to the other cars' preferences. Furthermore, there appear to be no mean differences in this population's preferences, since all estimated means are significantly equal to zero.

For this small problem, we have computed the ranking patterns' expected probabilities using (19) in Appendix A and obtained the $X_{2}$ and $G_{2}$ statistics to assess the absolute goodness of fit of the model. These statistics are also included in Table 3. As can be seen in this table, the limited and full information goodness of fit statistics agree that the unrestricted model cannot be rejected at $\alpha=0.01$. The ranking patterns' expected probabilities are given in Table 1 . A residual analysis using these probabilities revealed that the UMD solution provides a uniformly adequate fit to these data, whereas the WMD solution showed a large standardized residual (2.458) for the ranking pattern $\{1,2,4,3\}$. This is because objects $\{1,2,3\}$ are not together in this pattern and the model does not assign it a high probability, yet this pattern has one of the largest observed frequencies.

To illustrate how restricted Thurstonian models can be estimated in this framework we shall fit a one-factor model to these data. This is accomplished by fitting the following mean and correlation structure in the second stage: $\mu_{z^{*}}=\mathbf{D A} \mu_{t}$ and $\mathbf{P}_{z^{*}}=\mathbf{D A}\left(\lambda \lambda^{\prime}+\Psi\right) \mathbf{A}^{\prime} \mathbf{D}$. The identification restriction $\lambda_{4}=0$ is consistent with the estimation results in Table 2 , in which $\rho_{41}$ 
TABLE 3.

Results of estimating a one factor model model

Parameter estimates and asymptotic standard errors

\begin{tabular}{lcccccc} 
Estimator & $\lambda_{1}$ & $\lambda_{2}$ & $\lambda_{3}$ & $\psi_{1}$ & $\psi_{2}$ & $\psi_{3}$ \\
\hline WMD & 0.87 & 0.72 & 0.59 & 0.24 & 0.48 & 0.65 \\
& $(0.07)$ & $(0.09)$ & $(0.10)$ & $(0.11)$ & $(0.12)$ & $(0.11)$ \\
UMD & 0.81 & 0.72 & 0.58 & 0.35 & 0.48 & 0.66 \\
& $(0.08)$ & $(0.09)$ & $(0.11)$ & $(0.13)$ & $(0.13)$ & $(0.13)$ \\
\hline
\end{tabular}

Goodness of fit indices

\begin{tabular}{|c|c|c|c|c|c|c|}
\hline \multirow[b]{2}{*}{ Estimator } & \multicolumn{2}{|c|}{$H_{0}:\left\{\begin{array}{l}\boldsymbol{\mu}_{z^{*}}=\boldsymbol{\mu}_{z^{*}}(\theta) \\
\mathbf{P}_{z^{*}}=\mathbf{P}_{z^{*}}(\theta)\end{array}\right.$} & \multicolumn{2}{|c|}{$H_{0}: \pi=\pi(\theta)$} & $d f=20$ & \multirow[b]{2}{*}{$p$-value } \\
\hline & Test statistic & $p$-value & $X^{2}$ statistic & $p$-value & $G^{2}$ statistic & \\
\hline WMD & $N \hat{F}=24.96$ & 0.04 & 33.11 & 0.03 & 33.08 & 0.03 \\
\hline UMD & $\begin{array}{l}N \hat{F}=35.19 \\
T_{s}=18.80 \\
T_{a}=9.22\end{array}$ & $\begin{array}{l}-\overline{0.17} \\
0.23\end{array}$ & 31.85 & 0.05 & 32.83 & 0.04 \\
\hline
\end{tabular}

Notes: In this model we set $\mu_{t}=\mathbf{0}$. Also, for identification purposes, $\lambda_{4}=0$ and $\boldsymbol{\Psi}=\mathbf{I}-\operatorname{diag}\left(\lambda \lambda^{\prime}\right) . T_{a}$ and $T_{s}$ are Satorra and Bentler's (1988) adjusted and scaled test statistics. The degrees of freedom for $T_{a}$ are 6.87 .

and $\rho_{42}$ were significantly equal to zero. Also, as suggested by the results shown in Table 2, we shall set $\mu_{t}=\mathbf{0}$. The parameter estimates, asymptotic standard errors and goodness of fit tests of this model are shown in Table 3. As can be seen in this table, this model cannot be rejected at $\alpha=0.01$ either. However, the model slightly underpredicts (standardized residuals of 2.25 using UMD and 2.45 using WMD) the occurrence of the ranking pattern $\{1,2,4,3\}$.

\section{Discussion}

The limited information estimation approach adopted here bears some resemblance to Chan and Bentler's (1998) because both are two-stage. Note however that the reduced-form parameters of the model are estimated in our first stage, whereas the unrestricted Thurstonian parameters are estimated in Chan and Bentler's first stage.

In addition, our estimation approach to Thurstonian ranking models differ from Brady's (1989) and Chan and Bentler's (1998) in one key aspect. Brady estimated ranking models using the information contained in either binary or trinary rankings, whereas Chan and Bentler (1998) estimated ranking models using the information contained in those trinary rankings involving the object ranked first. Here, Thurstonian ranking models are estimated from the first and second order marginals of a $2^{\tilde{n}}$ contingency table which corresponds to binary, trinary and tetrad choice proportions. In Appendix $C$ we show that these proportions do not completely overlap and as a result our approach uses more information from the observed ranking patterns than either Brady or Chan and Bentler's approaches. An obvious drawback is that the dimension of the sample statistics used in our procedure is the largest of the three limited information approaches, being Chan and Bentler's the smallest. Aș a consequence, although our approach can handle larger models than those that can be estimated by full information maximum likelihood, it cannot be employed for models larger than say $n=10$.

The formulation of Thurstonian ranking models via (12) and (13) turns an idiosyncratic estimation problem into a mean and covariance structures with dichotomous indicators estimation problem. Well-known standard solutions for the latter (e.g., Muthén, 1978, 1984; see also 
Küsters, 1987) can be readily applied to the specific problem of fitting Thurstonian models to ranking data. The first stage estimators in Muthén (1978) and Muthén (1984) are obviously equivalent when applied to this problem, but the expressions for $\boldsymbol{\Gamma}$ differ since they build on different principles. In this paper we used the conceptually simpler approach of Muthén (1978) to obtain $\Gamma$, but Küsters' (1987) or Muthén's (1984) expressions could have been used instead.

An obvious advantage of our estimation approach is that under multinomial sampling of the ranking patterns Thurstonian models can be fitted using existing general purpose software for mean and covariance structure analysis. No special purpose computer program is required. However, the two most popular programs for covariance structure analysis, LISREL (Jöreskog \& Sörbom, 1993) and EQS (Bentler, 1995) cannot currently be used to fit Thurstonian ranking models since they implement correlation structure analysis for binary dependent variables, but not mean and correlation structure analysis (see Lee, Poon \& Bentler, 1995; Jöreskog, 1994) as required for Thurstonian modeling of ranking data. LISCOMP (Muthén, 1987) performs mean and correlation structure analysis of binary dichotomous variables using Muthén's (1978) estimator. Yet, the current version of LISCOMP cannot enforce the non-linear parameter constraints required to fit these models. Nonetheless, Thurstonian ranking models can be straightforwardly fitted using MECOSA (Arminger, Wittenberg \& Schepers, 1996). This program implements the WMD estimator using Küsters' (1987) expression for the asymptotic covariance matrix of the reduced form parameters. MECOSA can be used of course to obtain UMD parameter estimates, but does not currently provide correct standard errors and goodness of fit tests for the UMD estimator.

We have fitted an unrestricted Thurstonian model to the compact cars' data using full information maximum likelihood and the WMD estimator as implemented in MECOSA to illustrate our present discussion. In Table 4 we list the resulting parameter estimates, asymptotic standard

TABLE 4.

Results of estimating the unrestricted Thurstonian model using MECOSA and full information ML estimation

Parameter estimates and asymptotic standard errors

\begin{tabular}{lccccc}
$\mathbf{P}_{t}$ & Ford & $\begin{array}{c}\text { Opel } \\
\text { Corsa }\end{array}$ & $\begin{array}{c}\text { Peugeot } \\
106\end{array}$ & $\begin{array}{c}\text { Volkswagen } \\
\text { Polo }\end{array}$ & $\boldsymbol{\mu}_{t}$ \\
\hline Ford & 1 & 0.66 & 0.59 & 0.19 & 0.14 \\
Fiesta & & $(0.07)$ & $(0.08)$ & $(0.09)$ & $(0.09)$ \\
Opel & 0.66 & 1 & 0.47 & 0.12 & 0.10 \\
Corsa & $(0.07)$ & & $(0.09)$ & $(0.10)$ & $(0.09)$ \\
Peugeot & 0.50 & 0.44 & 1 & 0 & 0.08 \\
106 & $(0.09)$ & $(0.10)$ & & fixed & $(0.09)$ \\
Volkswagen & 0.16 & 0.13 & 0 & 1 & 0 \\
Polo & $(0.10)$ & $(0.11)$ & fixed & & fixed \\
\hline $\boldsymbol{\mu}_{t}$ & 0.16 & 0.12 & 0.09 & 0 & \\
& $(0.09)$ & $(0.09)$ & $(0.09)$ & fixed & \\
\hline
\end{tabular}

Goodness of fit indices

\begin{tabular}{|c|c|c|c|c|c|c|}
\hline \multirow[b]{2}{*}{ Estimator } & \multicolumn{2}{|c|}{$H_{0}:\left\{\begin{array}{l}\mu_{z^{*}}=\mu_{z^{*}}(\boldsymbol{\theta}) \\
\mathbf{P}_{z^{*}}=\mathbf{P}_{z^{*}}(\boldsymbol{\theta})\end{array}\right.$} & \multicolumn{2}{|c|}{$H_{0}: \pi=\pi(\theta)$} & $d f=15$ & \multirow[b]{2}{*}{$p$-value } \\
\hline & Test statistic & $p$-value & $X^{2}$ statistic & $p$-value & $G^{2}$ statistic & \\
\hline MECOSA & $N \hat{F}=17.13$ & 0.05 & 29.19 & 0.02 & 28.42 & 0.02 \\
\hline ML & - & - & 25.74 & 0.04 & 26.67 & 0.03 \\
\hline
\end{tabular}

Notes: MECOSA WMD estimates above the diagonal; full information ML estimates below the diagonal. The author is indebted to Ulf Böckenholt for providing the ML estimates. 
errors and goodness of fit tests, which are to be compared with those appearing in Table 2. As expected, the UMD parameter estimates are closer than the WMD estimates to the ML results. In fact, the UMD parameter estimates, standard errors and goodness of fit results are very close to those obtained by ML. For our small model, the results obtained using WMD are not too far from those obtained by ML, however. Furthermore, the differences between both WMD estimators are rather small.

An additional advantage of the present approach is that in almost all ranking applications, researchers are keenly interested in modeling additional information about the subjects' and/or objects' characteristics, particularly when the relevant characteristics of the objects to be compared are the result of some factorial experimental design (Böckenholt, 1992). This can be readily done within the mean and covariance structure analysis approach adopted here (see Muthén, 1982, 1984; Küsters, 1987), again using general-purpose software.

\section{Appendix A}

Alternative Expressions for Thurstonian Ranking Models as Functions of Differences of Normal Variates

Let $\mathbf{t}_{c}=\left(t_{r_{1}}, t_{r_{2}}, \ldots, t_{r_{n-1}}, t_{r_{n}}\right)^{\prime}$ be a vector of unobserved continuous preferences permuted according to ranking pattern $c$ and let $\mathbf{C}$ be the $(n-1)$ by $n$ matrix of contrasts

$$
\mathbf{C}=\left(\begin{array}{rrrrrr}
1 & -1 & 0 & \ldots & 0 & 0 \\
0 & 1 & -1 & \ldots & 0 & 0 \\
\ldots & \ldots & \ldots & \ldots & \ldots & \ldots \\
0 & 0 & 0 & \ldots & 1 & -1
\end{array}\right)
$$

After performing the linear transformation $\tilde{\mathbf{y}}^{*}=\mathbf{C t}_{c}$ we have

$$
\operatorname{Pr}\left(\mathbf{y}_{c}\right)=\operatorname{Pr}\left\{\left(\tilde{y}_{1}>0\right) \cap\left(\tilde{y}_{2}>0\right) \cap \cdots \cap\left(\tilde{y}_{n-1}>0\right)\right\}=\int_{0}^{\infty} \cdots \int_{0}^{\infty} \phi\left(\tilde{\mathbf{y}}^{*}: \mathbf{C} \boldsymbol{\mu}_{\tilde{t}}, \mathbf{C} \boldsymbol{\Sigma}_{\tilde{t}} \mathbf{C}^{\prime}\right) d \tilde{\mathbf{y}}^{*}
$$

Note that in (19) the contrast matrix $\mathbf{C}$ and the integration intervals are fixed for all ranking patterns, but the variables $t$ are permuted according to the ranking pattern. In contrast, in (12), ranking probabilities are obtained by changing the area of integration while the ordering of the variables $\mathbf{t}$ is fixed for all ranking patterns, $\mathbf{t}=\left(t_{o_{1}}, t_{o_{2}}, \ldots, t_{o_{n-1}}, t_{o_{n}}\right)^{\prime}$, and so is the contrast matrix A. Yao and Böckenholt (1999) expressed Thurstonian ranking models using (19).

An alternative expression for Thurstonian ranking models is obviously obtained by leaving the ordering of the variables $t$ intact and the integration intervals fixed for all ranking patterns but changing the contrast matrix according to the rank order. In that case

$$
\operatorname{Pr}\left(\mathbf{y}_{c}\right)=\int_{0}^{\infty} \cdots \int_{0}^{\infty} \phi\left(\mathbf{y}_{c}^{*}: \mathbf{A}_{c} \boldsymbol{\mu}_{t}, \mathbf{A}_{c} \boldsymbol{\Sigma}_{t} \mathbf{A}_{c}^{\prime}\right) d \mathbf{y}_{c}^{*}
$$

where we use $\mathbf{A}_{c}$ to denote that the contrast matrix depends on the rank order $\mathbf{r}_{\boldsymbol{c}}$. If we standardize $\mathbf{y}_{c}^{*}$ using $\mathbf{z}_{c}^{*}=\mathbf{D}_{c} \mathbf{y}_{c}^{*}$, where $\mathbf{D}_{c}=\operatorname{diag}\left(\mathbf{A}_{c} \boldsymbol{\Sigma}_{t} \mathbf{A}_{c}^{\prime}\right)^{-1 / 2}$, we obtain

$$
\operatorname{Pr}\left(\mathbf{y}_{c}\right)=\Phi_{n-1}\left(\mathbf{z}_{c}^{*}: \mathbf{0}, \mathbf{D}_{\mathbf{c}} \mathbf{A}_{\mathbf{c}} \boldsymbol{\Sigma}_{\mathbf{t}} \mathbf{A}_{\mathbf{c}}^{\prime} \mathbf{D}_{\mathbf{c}}\right)
$$

a $n-1$ dimensional cumulative standard normal distribution to be evaluated at $\mathbf{D}_{c} \mathbf{A}_{c} \boldsymbol{\mu}_{t}$. Both Böckenholt (1992) and Chan and Bentler (1998) used (21) to portray Thurstonian ranking models. 
Appendix B

\section{Alternative Identification Restrictions for the Unrestricted Thurstonian Ranking Model}

For the parameters of the Thurstonian model to be identifiable from their reduced form parameters, the system of linear equations $\mathbf{y}^{*}=\mathbf{A t}$ must have a solution. However, $\mathbf{A}$ is of rank $n-1$ and therefore, the parameters of the unrestricted model for ranking data bear a location indeterminacy. One way of solving this indeterminacy is by reparameterization: $\mathbf{A}$ can be factored as $\mathbf{A}=\mathbf{K S}$, where $\mathbf{S}$ is an $(n-1) \times n$ matrix and $\mathbf{K}$ is an $\tilde{n} \times(n-1)$ matrix. $\mathbf{S}$ may be arbitrarily chosen provided that its rows are in the row space of $\mathbf{A}$ (Bock, 1975). Then $\mathbf{K}$ can be obtained as $\mathbf{K}=\mathbf{A} \mathbf{S}^{\prime}\left(\mathbf{S S}^{\prime}\right)^{-1}$. A matrix $\mathbf{S}$ that obviously satisfies the above condition is the reduced rowechelon form of $\mathbf{A}, \mathbf{S}=\left[\mathbf{I}_{n-1} \mid-\mathbf{l}_{n-1}\right]$. With this choice of $\mathbf{S}, \mathbf{K}$ equals the first $n-1$ columns of $\mathbf{A}$. Using this factorization of $\mathbf{A}$ we reparameterize the unrestricted Thurstonian model for ranking data as $\mathbf{y}^{*}=\mathbf{K z}$, where $\mathbf{z}=\mathbf{S t}$. With this reparameterization, the parameters being estimated are

$$
\boldsymbol{\mu}_{z}=\mathbf{S} \boldsymbol{\mu}_{t} \quad \text { and } \quad \boldsymbol{\Sigma}_{z}=\mathbf{S} \Sigma_{t} \mathbf{S}^{\prime}
$$

Naturally, as in (15), a variance normalization restriction on $\mathbf{y}^{*}$ must be enforced by $\mathbf{z}^{*}=\mathbf{D y ^ { * }}$, and one parameter must be fixed in order to account for the model insensitivity to scale changes in $\mathbf{t}$ of the type $\mathbf{t}^{*}=c \mathbf{t}$. Arbitrarily, we could set $\sigma_{z_{1}}^{2}=1$. These identification restrictions were employed by Maydeu-Olivares (1995). Of course, different choices of the basis matrix $\mathbf{S}$ lead to different reparameterizations. For instance, Chan and Bentler (1998) estimated the unrestricted Thurstonian ranking model letting $\mathbf{S}=\left[\mathbf{I}_{n-1} \mid-\mathbf{I}_{n-1}\right]$.

Reparameterization is just one method to solve a system of linear equations of deficient rank such as $\mathbf{y}^{*}=\mathbf{A t}$. An alternative approach is by imposing an additional restriction that it is not in the row space of $\mathbf{A}$. One restriction that satisfies this condition is

$$
\mathbf{l}_{n-1}^{\prime} \mathbf{t}_{n-1}=t_{n}
$$

where $\mathbf{t}_{n-1}$ denotes the first $(n-1)$ unobserved continuous preferences, and $t_{n}$ denotes the last one. Now, partition the parameters of the unrestricted Thurstonian model for ranking data according to the partitioning of $\mathbf{t}_{n}=\left(\mathbf{t}_{n-1}, t_{n}\right)^{\prime}, \boldsymbol{\mu}_{t}=\left(\begin{array}{l}\boldsymbol{\mu}_{1} \\ \boldsymbol{\mu}_{2}\end{array}\right)$ and $\boldsymbol{\Sigma}_{t}=\left(\begin{array}{ll}\boldsymbol{\Sigma}_{11} & \boldsymbol{\Sigma}_{21}^{\prime} \\ \boldsymbol{\Sigma}_{21} & \boldsymbol{\Sigma}_{22}\end{array}\right)$, where $\mu_{2}=\mu_{n}, \Sigma_{22}=\sigma_{n}^{2}$, and $\Sigma_{21}=\left(\sigma_{21}, \sigma_{31}, \ldots \sigma_{n 1}\right)^{\prime}$. Then, from (23) we obtain the following set of restrictions among the parameters of the model

$$
\mu_{2}=\mathbf{l}_{n-1}^{\prime} \mu_{1}, \quad \Sigma_{21}=\mathbf{l}_{n-1}^{\prime} \Sigma_{11}, \quad \text { and } \quad \Sigma_{22}=\mathbf{l}_{n-1}^{\prime} \Sigma_{11} \mathbf{l}_{n-1}
$$

Hence, the unrestricted Thurstonian model for ranking data can be parameterized as $\mathbf{y}^{*}=\mathbf{A t}$, provided that the parameters of the model are subject to restrictions of the type (24). Again, to identify the model we perform the transformation $\mathbf{z}^{*}=D \mathbf{y}^{*}$, and we fix one parameter to account for the model insensitivity to scale changes in $t$ of the type $t^{*}=c t$.

In this identification solution by restrictions in the unknowns, the "true" parameters of the model, $\mu_{t}^{0}$ and $\Sigma_{t}^{0}$, are expressed as a function of $\mu_{1}$ and $\Sigma_{11}$ as follows: Partition $\mu_{t}^{0}$ and $\Sigma_{t}^{0}$ as above with (24). Accordingly, partition $\mathbf{A}$ as $\mathbf{A}=\left(\mathbf{A}_{1}, \mathbf{A}_{2}\right)$ where $\mathbf{A}_{1}$ and $\mathbf{A}_{2}$ are the first $n-1$ and last columns of $\mathbf{A}$, respectively. Now let $\mathbf{E}=\left(\mathbf{A}_{1}+\mathbf{A}_{2} \mathbf{l}_{n-1}^{\prime}\right)$ a $\tilde{\boldsymbol{\mu}} \times(n-1)$ matrix of full column rank with a $g$-inverse $\mathbf{E}^{-}$. Then,

$$
\begin{aligned}
\boldsymbol{\mu}_{1} & =\mathbf{E}^{-} \mathbf{A} \boldsymbol{\mu}_{t}^{0} \\
\boldsymbol{\Sigma}_{11} & =\mathbf{E}^{-} \mathbf{A} \Sigma_{t}^{0} \mathbf{A}^{\prime} \mathbf{E}^{-\prime}
\end{aligned}
$$

Consequently, this parameterization of the model can be alternatively written as $\mathbf{y}^{*}=\mathbf{E} \mathbf{t}_{n-l}$. 
That is,

$$
\begin{aligned}
\boldsymbol{\mu}_{y}^{*} & =\mathbf{E} \boldsymbol{\mu}_{1} \\
\boldsymbol{\Sigma}_{y^{*}} & =\mathbf{E} \boldsymbol{\Sigma}_{11} \mathbf{E} .
\end{aligned}
$$

The identification solution by restrictions in the unknowns has the advantage over the identification solution given in section 4 that it provides an estimate of the covariance matrix of the unobserved continuous preferences instead of its correlation matrix. However, this covariance matrix is not unique since it depends on the choice of the restriction (23). The identification solution by reparameterization besides being non unique due to the arbitrary choice of basis matrix has the disadvantage that the reparameterized parameters are hardly interpretable.

\section{Appendix C \\ Asymptotic Distribution of Muthén's (1978) First Stage Estimator in Ranking Data}

Consider an $m$-dimensional vector of dichotomous random variables $\mathbf{y}$, where $y_{l}=$ $\{0,1\} \forall l$. Given a random sample of size $N$, let $p_{l}$ and $p_{l l^{\prime}}$ be the sample counterparts of $\pi_{l}=$ $\operatorname{Pr}\left(y_{l}=1\right)$ and $\pi_{l l^{\prime}}=\operatorname{Pr}\left(y_{l}=1, y_{l^{\prime}}=1\right), l=2, \ldots, m ; l^{\prime}=1, \ldots, m-1$. Construct $\dot{\mathbf{p}}=\left(\begin{array}{c}\mathbf{p}_{1} \\ \mathbf{p}_{2}\end{array}\right)$ where $\mathbf{p}_{1}^{\prime}=\left(p_{1}, p_{2}, \ldots, p_{m}\right)$ and $\mathbf{p}_{2}^{\prime}=\left(p_{21}, p_{31}, \ldots, p_{m, m-1}\right)$ are the (nonredundant) first and second order marginals of the $2^{m}$ contingency table.

Suppose $\mathbf{y}$ arises from the dichotomization of a normal density

$$
\pi_{c}=\operatorname{Pr}\left(\mathbf{y}_{c}\right)=\int \dddot{R} \int \phi\left(\mathbf{z}^{*}: \mu_{z^{*}}, \mathbf{P}_{z^{*}}\right) d \mathbf{z}^{*}, \quad c=1, \ldots, 2^{m}
$$

where $\mathbf{R}$ is a parallelepiped defined by (27). Letting $\mu_{z^{*}}=-\tau$ in (23), Muthén (1978) showed that since $\hat{\boldsymbol{\kappa}}=\left(\begin{array}{c}\boldsymbol{\mu}_{z^{*}} \\ \operatorname{vecr}\left(\hat{\mathbf{P}}_{z^{*}}\right)\end{array}\right)$ is a one-to-one nonlinear transformation of $\dot{\mathbf{p}}$,

$$
\sqrt{N}\left(\hat{\boldsymbol{\kappa}}-\kappa_{0}\right) \stackrel{d}{\rightarrow} N\left(\mathbf{O}, \mathbf{\Gamma}=\mathbf{\Delta} \mathbf{\Omega} \boldsymbol{\Delta}^{\prime}\right),
$$

where $\boldsymbol{\Delta}=\left(\frac{\partial \dot{\boldsymbol{\pi}}}{\partial \boldsymbol{\kappa}^{\prime}}\right)_{\kappa=\kappa_{0}}^{-1}$, and $\boldsymbol{\Omega}$ is the asymptotic covariance matrix of $\sqrt{N}\left(\dot{\mathbf{p}}-\dot{\pi}_{0}\right)$.

When the dichotomous patterns $\mathbf{y}$ arise from a ranking experiment, $m=\tilde{n}$, and the $2^{\tilde{n}}$ contingency table (and hence $\mathbf{p}$ ) has $2^{\tilde{n}}-n$ ! structural zeroes. We wish to (a) show that $\hat{\boldsymbol{\kappa}}$ is still asymptotically normal in this case, and (b) determine the rank of $\Gamma$.

To do so, first consider $\mathbf{Y}^{\circ}$, a $2^{m} \times m$ matrix containing all possible binary patterns, and let $\mathbf{p}$ be its associated vector of observed proportions. Construct a $m+\frac{m(m-1)}{2} \times 2^{m}$ matrix $\mathbf{H}=\left(\begin{array}{l}\mathbf{H}_{1} \\ \mathbf{H}_{2}\end{array}\right)$, partitioned according to $\dot{\mathbf{p}}$, as

$$
\begin{array}{rlrl}
\mathbf{h}_{l}^{\prime} & =\mathbf{y}_{l}^{\prime}, \forall \mathbf{h}_{l}^{\prime} \in \mathbf{H}_{1} & l & =1, \ldots, m \\
\mathbf{h}_{l, l^{\prime}}^{\prime} & =\mathbf{y}_{l}^{\prime} \odot \mathbf{y}_{l^{\prime}}^{\prime}, \forall \mathbf{h}_{l, l^{\prime}}^{\prime} \in \mathbf{H}_{2}, & & l=2, \ldots, m ; l^{\prime}=1, \ldots, m-1
\end{array}
$$

where $\odot$ denotes an Hadamard product. Direct verification shows that

$$
\dot{\mathbf{p}}=\mathbf{H p} \text {. }
$$

Suppose now that the observed dichotomous patterns $\mathbf{y}$ arise from a ranking experiment. Let $\mathbf{p}_{r}$ be an $n$ !-dimensional vector of sample proportions associated with the observed ranking patterns. Under multinomial sampling $\sqrt{N}\left(\mathbf{p}_{r}-\boldsymbol{\pi}_{r}\right) \stackrel{d}{\rightarrow} N(\mathbf{O}, \Xi)$ with $\Xi=\operatorname{diag}\left(\boldsymbol{\pi}_{r}\right)-\boldsymbol{\pi}_{r}^{\prime} \boldsymbol{\pi}_{r}$. 
Since $\mathbf{p}=\mathbf{T} \mathbf{p}_{r}$, where $\mathbf{T}$ is a $2^{\tilde{n}} \times n !$ augmentation matrix with $\{0,1\}$ entries in appropriate positions, it follows from (25) and (27) that

$$
\sqrt{N}\left(\hat{\boldsymbol{\kappa}}-\boldsymbol{\kappa}_{0}\right) \stackrel{d}{\rightarrow} N\left(\mathbf{0}, \boldsymbol{\Gamma}=\Delta \mathbf{H T} \Xi^{\prime} \mathbf{T}^{\prime} \mathbf{H}^{\prime} \Delta^{\prime}\right)
$$

The rank of $\boldsymbol{\Gamma}$ can be determined from (31) by noticing the following: First, $\Delta$ is lower-triangular (Muthén, 1978: p. 554), and hence nonsingular under the usual regularity conditions for $\pi_{r}$. Hence, the rank of $\Gamma$ equals the rank of $\Omega$. Second, $\mathbf{H}^{\bullet}=\mathbf{H T}$ can be obtained from $\mathbf{Y}^{\bullet}$ directly using (29), where $\mathrm{Y}^{\bullet}$ is an $n ! \times \tilde{n}$ matrix containing all possible ranking patterns in binary form. (For $n=4, \mathbf{Y}^{\bullet}$ appears in Table 1). Third, $\Omega=\mathbf{H}^{\bullet} \boldsymbol{\Xi} \mathbf{H}^{\bullet \prime}=\mathbf{H}^{\bullet} \boldsymbol{\Xi}_{n !-1} \mathbf{H}^{\bullet \prime}$, since the first column of $\mathbf{H}^{\bullet}$ is zero by construction, where $\boldsymbol{\Xi}_{n !-1}$ is the appropriate submatrix of $\boldsymbol{\Xi}$. Since $\boldsymbol{\Xi}_{n !-1}$ is of full rank by the multinomial restriction $\sum \pi_{r}=1, r(\boldsymbol{\Gamma})=r(\boldsymbol{\Omega})=r\left(\mathbf{H}^{\bullet}\right)$. Finally, the rank of $\mathbf{H}^{\bullet}$ can be easily verified to be given by (18) by mathematical induction after reducing this matrix to row echelon form using elementary row operations.

A consistent estimate of $\Gamma, \Gamma$, can be obtained from (31) by replacing $\mathbf{p}_{r}$ for $\pi_{r}$ to consistently estimate $\boldsymbol{\Omega}$, and evaluating $\Delta$ at $\kappa=\hat{\kappa}$. Similarly, a consistent estimate of $\boldsymbol{\Gamma}^{-}$can be obtained by a spectral decomposition of $\hat{\Gamma}$ since $v$ is known. In practice, there are computationally more efficient ways to estimate $\boldsymbol{\Omega}$. One of them is by using the explicit expression for the elements of $\boldsymbol{\Omega}$ given by Christoffersson (1975, Appendix 2 ). Another is directly from $\mathbf{Y}$, the $N \times 2^{m}$ raw data matrix, using $\hat{\mathbf{\Omega}}=\left(\mathbf{D}-\mathbf{l}^{\prime}\right)^{\prime}\left(\mathbf{D}-\overline{\mathbf{l}}^{\prime}\right) / N$, where $\mathbf{D}$ here denotes a $N \times \frac{m(m+1)}{2}$ matrix with $\mathbf{d}_{j}=\left(\operatorname{vecdiag}\left(\mathbf{y}_{j}^{\prime} \mathbf{y}_{j}\right)\right.$, vecr( $\left.\left(\mathbf{y}_{j}^{\prime} \mathbf{y}_{j}\right)\right), j=1, \ldots, N$, and $\overline{\mathbf{d}}^{\prime}=\mathbf{l}^{\prime} \mathbf{D} / n$.

In closing, we would like to note that the redundancies among the elements of $\dot{\mathbf{p}}$ in ranking data can be obtained by computing a basis for the nullspace of $\mathbf{H}^{\bullet}$ for instance via the singular value decomposition theorem. As an example, when $n=4$, we find that the $v=4$ redundancies are

$$
\begin{aligned}
& p_{54}-p_{64}-p_{5}+p_{65}=0 \\
& p_{32}-p_{62}-p_{3}+p_{63}=0 \\
& p_{31}-p_{51}-p_{3}+p_{53}=0 \\
& p_{21}-p_{41}-p_{2}+p_{42}=0
\end{aligned}
$$

References

Arminger, G., Wittenberg, J., \& Schepers, A. (1996). MECOSA 3. User guide. Friedrichsdorf: Additive GmbH.

Bekker, P.A., Merckens, A., \& Wansbeek, T.J. (1994). Identification, equivalent models and computer algebra. San Diego: Academic Press.

Bentler, P.M. (1995). EQS Structural Equations Program Manual. Encino, CA: Multivariate Software.

Bock, R.D. (1975). Multivariate statistical methods in behavioral research. New York: McGraw Hill.

Bock, R.D., \& Jones, L.V. (1968). The measurement and prediction of judgment and choice. San Francisco: Holden-Day.

Böckenholt, U. (1992). Thurstonian representation for partial ranking data. British Journal of Mathematical and Statistical Psychology, 45, 31-49.

Böckenholt, U. (1993). Applications of Thurstonian models to ranking data. In M.A. Fligner \& J.S. Verducci (Eds). Probability models and statistical analyses for ranking data. New York: Springer-Verlag.

Brady, H.E. (1989). Factor and ideal point analysis for interpersonally incomparable data. Psychometrika, 54, 181-202.

Chan, W., \& Bentler, P.M. (1998). Covariance structure analysis of ordinal ipsative data. Psychometrika, 63, 369-399.

Christoffersson, A. (1975). Factor analysis of dichotomized variables. Psychometrika, 40, 5-32.

Clark, T.E. (1996). Small-sample properties of estimators of nonlinear models of covariance structure. Journal of Bussiness and Economic Statistics, 14, 367-373.

Dansie, B.R. (1986). Normal order statistics as permutation probability models. Applied Statistics, 3, 269-275.

Jöreskog, K.G. (1994). On the estimation of polychoric correlations and their asymptotic covariance matrix. Psychometrika, 59, 381-389.

Jöreskog, K.G., \& Sörbom, D. (1993). LISREL 8. User's reference guide. Chicago, IL: Scientific Software.

Küsters, U.L. (1987). Hierarchische Mittelwert- und Kovarianztrukturmodelle mit nichtmetrischen endogenen Variablen [Hierarchical mean and covariance structure models on nonmetric endogenous variables]. Heidelberg: PhysicaVerlag.

Lee, S.Y., Poon, W.Y., \& Bentler, P.M. (1995). A two-stage estimation of structural equation models with continuous and polytomous variables. British Journal of Mathematical and Statistical Psychology, 48, 339-358. 
Maydeu-Olivares, A. (1995, July). Structural equation modeling of paired comparisons and ranking data. Paper presented at the 9th European Meeting of the Psychometric Society. Leiden, The Netherlands.

Muthén, B. (1978). Contributions to factor analysis of dichotomous variables. Psychometrika, 43, 551-560.

Muthén, B. (1982). Some categorical response models with continuous latent variables. In K.G. Jöreskog \& H. Wold (Eds.). Systems under indirect observation. (Vol 1). Amsterdam: North Holland.

Muthén, B. (1984). A general structural equation model with dichotomous, ordered categorical, and continuous latent variable indicators. Psychometrika, 49, 115-132.

Muthén, B. (1987). LISCOMP: Analysis of linear structural equations using a comprehensive measurement model. Mooresville, IN: Scientific Software.

Muthén, B. (1993). Goodness of fit with categorical and other non normal variables. In K.A. Bollen \& J.S. Long (Eds.) Testing structural equation models. Newbury Park, CA: Sage.

Muthén, B., \& Satorra, A. (1995). Technical aspects of Muthén's LISCOMP approach to estimation of latent variable relations with a comprehensive measurement model. Psychometrika, 60, 489-503.

Rao, C.R. (1973). Linear statistical inference and its applications (2nd ed). New York: Wiley.

Satorra, A. (1989). Alternative test criteria in covariance structure analysis: A unified approach. Psychometrika, 54, $131-151$

Satorra, A., \& Bentler, P.M. (1988). Scaling corrections for chi-square statistics in covariance structure analysis. ASA 1988 Proceedings of the Business and Statistics section, 308-313.

Takane, Y. (1987). Analysis of covariance structures and probabilistic binary choice data. Communication and Cognition, $20,45-62$.

Takane, Y. (1989). Analysis of covariance structures and probabilistic binary choice data. In G. Soete, H. Feger \& K.C. Klauer (Eds.), New developments in psychological choice modeling (pp. 139-160). Amsterdam: Elsevier Science.

Takane, Y., \& de Leeuw, J. (1987). On the relationship between item response theory and factor analysis of discretized variables. Psychometrika, 52, 393-408.

Thurstone, L.L. (1927). A law of comparative judgment. Psychological Review, 79, 281-299.

Thurstone, L.L. (1931). Rank order as a psychological method. Journal of Experimental Psychology, 14, 187-201.

Yao, K.G., \& Böckenholt, U. (1999). Bayesian estimation of Thurstonian ranking models based on the Gibbs sampler. British Journal of Mathematical and Statistical Psychology, 52, 79-92.

Manuscript received 3 FEB 1998

Final version received 2I SEPT 1998 